\title{
bei Kindern häufig falsch behandelt
}

\author{
Kinder und Jugendliche suchen ihre Grenzen beim Downhill-Biken, auf dem Snowboard oder beim \\ Trampolinspringen. Doch ausgerechnet bei diesen beliebten Trendsportarten kommt es häufig zu Unfällen \\ mit Gelenkverletzungen und Frakturen sowie zum Teil schweren Kopftraumen. Bei der Behandlung von \\ Knochenbrüchen im Wachstumsalter geschehen viele Fehler, wie Prof. Lucas Wessel von der Kinderchirurgi- \\ schen Klinik des Universitätsklinikums Mannheim weiß.
}

? Welche Sportarten bergen für Kinder und Jugendliche die höchsten Unfallgefahren?

Wessel: Die höchsten Unfallzahlen bei Kindern sehen wir beim Snowboarden, beim Skateboarden und Inline-Skaten sowie beim Trampolinspringen. Auch Mountainbiken, insbesondere Downhill, gilt als unfallträchtig, hier sind vorwiegend Jugendliche betroffen.

? Welche typischen Verletzungen treten bei Trendsportarten auf?

Wessel: Bei den Downhill-Bikern sind am häufigsten die oberen Extremitäten betroffen. Am schlimmsten sind allerdings die Kopfverletzungen infolge schwerer Stürze. Die Verletzungen beim Snowboarden und Inline-Skaten sind ähnlich. Meist leiden hier die Handgelenke. Bei Snowboardern kommen Verletzungen des Unterschenkels und der Sprunggelenke hinzu, da sie keine festen Stiefel, sondern Softboots tragen. Einer amerikanischen Studie zufolge erleiden bei Snowboard-Unfällen 27\% der Kinder Kopf- und Halsverletzungen, bei $58 \%$ sind die oberen und bei $10 \%$ die unteren Extremitäten betroffen. Ähnliche Blessuren, einschließlich Kopfverletzungen, entstehen auch bei dem neuerdings sehr beliebten Downhill-Skateboarden. Beim Trampolinspringen sind durch das Abfangen von Stürzen vor allem die oberen Extremitäten gefährdet. Besonders kritisch ist es, wenn Kinder unterschiedlichen Gewichts springen. Dann können die Kleinsten hinauskatapultiert werden, was schwere Kopfverletzungen zur Folge haben kann. Beim Klettern kommen überwiegend umgeknickte Finger oder Frakturen an den Extremitäten vor.

? Welche Vorsichtsmaßnahmen schützen vor diesen Verletzungen?

Wessel: Beim Snowboarden, Inline-Skatenund Skateboardfahren können Handgelenkschoner entsprechende Verletzungen verhindern. Vor schweren Kopfverletzungen kann nur ein Helm schützen, auch wenn er als uncool empfunden wird. Immer wieder wird hier, wie auch beim Reiten,

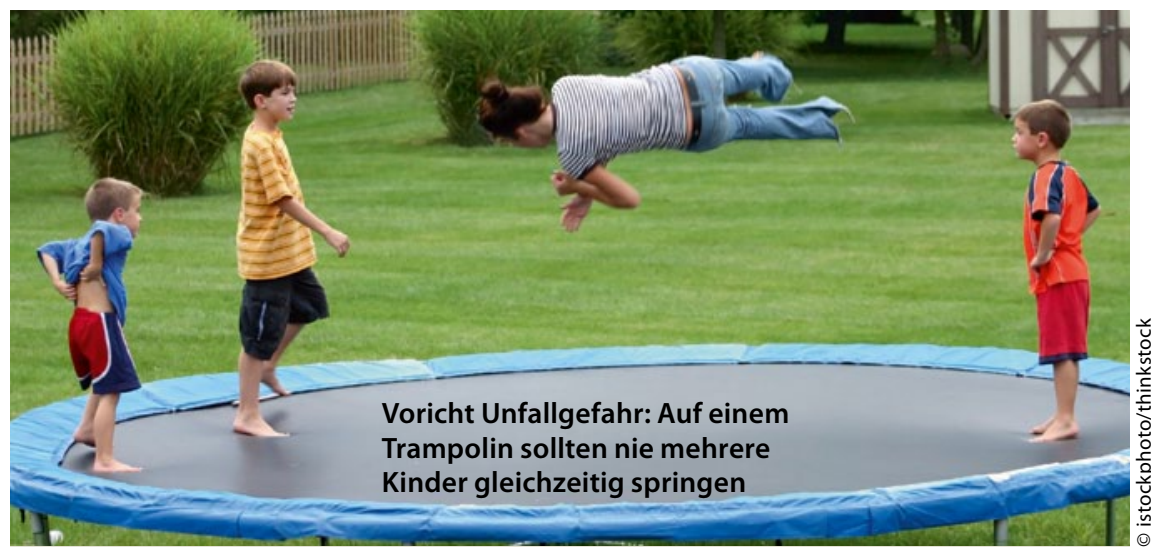

über die Vorteile von Rückenschonern diskutiert. Es gibt allerdings bislang keine Studie, die deren Nutzen belegt. Ein Trampolin wird mit einem Fangnetz sicherer. Auf keinen Fall sollten mehrere Kinder gleichzeitig springen, damit es nicht zu Zusammenstößen kommt.

? Bei der Behandlung von Kindern mit Unfallverletzungen wird über hohe Fehlerquoten berichtet. Was läuft da schief? Wessel: Jeder Chirurg darf in Deutschland Kinder behandeln. Das Problem ist aber, dass Unfallverletzungen bei Kindern oft anders therapiert werden müssen als bei Erwachsenen. Da sie noch im Wachstum sind, werden Fehlstellungen oft durch korrigierendes Wachstum spontan ausgeglichen. Behandlungsfehler geschehen vor allem an Oberarm und Handgelenk. Aus den Zahlen der Schlichtungsstellen der Deutschen Ärztekammern wird deutlich, dass die Binsenweisheit, dass Kinder keine kleinen Erwachsenen sind, zu selten beherzigt wird. Fehler betreffen eine ungenaue klinische Befunderhebung, die Fehldeutung von Röntgenbildern, eine dem Frakturmuster nicht angemessene konservative oder operative Therapie oder eine unterlassene beziehungsweise unzureichende Frakturkontrolle. Die Behandlung von Frakturen um das Ellenbogengelenk zeigte bei Schlichtungsverfahren mit $77 \%$ die höchste Fehlerquote. Hier wird z. B. viel zu häufig mit Plattenosteosynthesen gearbeitet, wenn ein einfacher Kirschner-Draht oder auch nur ein Gips genügen würde.

Interview: Dr. Christine Starostzik 